\title{
People - Space - Technology: An Ethnographic Approach
}

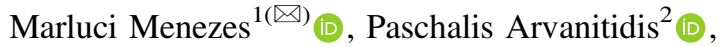 \\ Therese Kenna ${ }^{3}$ (1), and Petja Ivanova-Radovanova ${ }^{4}[$ \\ ${ }^{1}$ National Laboratory for Civil Engineering - LNEC, Lisbon, Portugal \\ marluci@lnec.pt \\ 2 Department of Economics, University of Thessaly, Volos, Greece \\ parvanit@uth.gr \\ ${ }^{3}$ Department of Geography, University College Cork, Cork, Ireland \\ t.kenna@ucc.ie \\ 4 Association for Integrated Development and Sustainability, Sofia, Bulgaria \\ petjaivanova@gmail.com
}

\begin{abstract}
CyberParks aims at advancing knowledge on the relationship between information and communication technologies and the socially sustainable production and usage of public open spaces. Such research necessitates a solid methodological base. Urban ethnography brings together a number of perspectives and approaches to deal with cultural and social aspects of urban life, and as such it is able to provide an integrated methodological framework for the study of technology-public space relationship. The ethnographic approach means, by definition, an in-depth, micro-scale look at the phenomena under concern. However, the technological dimension makes the relationship between people and space more complex. This is not simply because an additional layer of analysis is added; it comes as a result of the emergence of multiple connections between the real and the virtual. From an ethnographic perspective, this requires the researcher to capture, explore and understand the cyber-social phenomena and dynamics in a multifaceted, hybrid, triangulated and crossreferenced way. This makes ethnographic research much more complicated but more interesting as well. The current chapter attempts to outline such an analytical framework to guide empirical research on the issues. This framework draws on the public space literature and adds the technological dimension brought in by the CyberParks project. We argue that this enriches the ethnographic approach providing a more integrated framework for the analysis of the relationship between people, space and technology.
\end{abstract}

Keywords: Ethnographic perspective - Methodological framework · Micro-scale $\cdot$ Social hybrid dynamics $\cdot$ Cyber phenomena 


\section{Introduction: Capturing the Social Cyber Phenomenon in Urban Public Spaces}

The improvement, through Information and Communication Technologies (ICT), of the quality of urban life, the inclusion and social participation in the design of public open spaces (POS), and the development of tools for studying and supporting urban planning, constitute basic pursuits of the CyberParks project. Certainly, all these presuppose and necessitate a clear and coherent view of the relationship between people, space and technology, and the dynamics of their links. Questions that come to the fore include: What kind of public spaces, people, practices and needs are we talking about? How does the introduction of ICT affect both space and people? How are POS design, planning and usage affected by the osmosis of the real with the virtual? What kind of new experiences, discourses, practices and expectations would emerge in such world?

Ethnography is a methodological perspective that can shed light on all these questions. This is due to its ability to explore "understudied regions of society" and to "illuminate the unknown" (cf. Didier Fassin, in Low 2016: 2), placing emphasis on people and their preferences, practices and experiences. Such oriented ethnography can provide a base to the study of socio-spatial questions, and the development of knowledge about the people-space-technology relationship. In that direction, we follow Low (2016) to conceive space as a site of culture, to draw on the notion of spatialization $^{1}$ that takes under proper consideration the multiple, translocal and unequal social processes of production and construction of space $^{2}$, and to use ethnography as an approach for the understanding of people's living within space.

The notion of spatialization of culture (Low 2016) allows us to consider that people create spaces through their bodies and behaviours, discourses, emotive and affective aspects, as well as through their mobility and trajectories. Space is thus embodied and endowed with meanings, just as everyday patterns, movements, and trajectories occur in specific places and landscapes. Thus, ethnography of socio-spatial questions is oriented towards capturing place making. In summary, these issues contribute to the reciprocal incorporation of the material and the experiential.

However, constituting ICT as a tangible dimension of the social (Castells 2001) leads to the emergence of complex socio-spatial experiences, generating a multiplicity of connections (and disconnections) between real-real and virtual-real. This is not simply because an additional layer of analysis in added, giving rise to a dualism between the real and the virtual. In fact, the complexity is the result of the emergence of new relationships between the real-real and the real-virtual, in which cybernetic reality increasingly becomes a tangible dimension of the social. From an ethnographic perspective, this requires the researcher to capture, explore and understand the cyber-social

\footnotetext{
${ }^{1}$ By spatialization we mean the physical, historical, affective and discursive production and localization process of social relations, institutions, representations and practices in space.

${ }^{2}$ By social production of space, we consider the factors - social, economic, ideological and technological - that contribute to the creation and materialization of a setting. The social construction of space is here considered as the phenomenological and symbolic experiences that take place in space, being mediated by the change, conflict and control process (Low 2000a, b).
} 
phenomena and dynamics in a multifaceted, hybrid, triangulated and cross-referenced way. It should be pointed out, however, that the methodological perspective advocated here is not ethnography of the virtual space. It is rather an approach to capture the richness of the relationship between people, space and ICT. In other words, we are interested here in an ethnographic analysis of socio-spatial issues related to urban public spaces, taking into proper account the technological element and the changes it leads to. That is, in particular, it is intended to finding ways to help answer questions, such as: What is known about the relationship between new media use and spatial practices? What do people want from an ICT enhanced public open space? How do these requirements differ by socioeconomic status, gender and age? What technological developments are most likely to enhance current user behaviour or develop new user behaviours?

On these grounds, the current chapter provides introductory methodological and applied guidance for doing ethnography with a focus on the triptych people-spacetechnology. In achieving this objective, the arguments presented here place emphasis on the following aspects: (1) the ethnographic issues that the CyberParks project presents and the challenges they put to the scholar of the contemporary urban phenomenon; and synthetic explanation of what an ethnographic approach means, emphasizing the importance of capturing the phenomena under study from a microscale and a close and deep perspective; (2) an ethnographic analytical perspective seen through the lenses and objectives set by the CyberParks project, namely: to bring more people into POS; suggest urban design responses that are closer to people's needs and interests; increase people's participation in the design of POS; improve the planning and urban design processes; and to develop an ethnographic analytical framework on the main dimensions about which specific studies will be carried out.

\section{Ethnographic Perspective to Capture the Cyberpark Phenomenon}

The urban ethnographic perspective to CyberParks analysis aims to advance understanding of the relation between space-people-technology and its dynamics, through cultural and sociological fieldwork and by employing various theoretical and methodological approaches. In particular, it brings together knowledge about the use of new ICT in POS, enhances understanding of POS and of human behaviour in the context of new media, and offers advice for appropriate ways in employing technology within public spaces. To do so, researchers should observe uses of technology within space as well as behaviours in space of all people, including those using and those not using technology. But in what sense can an ethnographic perspective help us to clarify and understand the people-space relationship and its links with technology?

The first aspect to consider is the unquestionable influence of ICT in the growing, accelerated and unequal transformation of society and, as such, of urban contexts. What, in the first instance, hinders the design of public spaces in a manner that is more inclusive, participative, healthy and connected with the needs and desires of the people? That is, there is a lack of knowledge about the relation between people-space-technology in order to think and to create POS that are more suitable to people, despite the fact that 
there is a proliferation of studies in the contemporary public space literature. This is because the majority of such studies are of a macro-analytical nature and, in many cases, the design of space is privileged to the detriment of the understanding of the relationship between people and space, even if digital technologies are included in this connection. What we argue is that in order to capture and better understand the socio-spatial issues with proper emphasis on the ICT dimension, it is important to carry out smaller, more intensive and comprehensive studies, and pervade by dense descriptions.

The above, we advocate, provides a more precise approximation and increases the possibility of producing useful practical (and theoretical) knowledge of the relationship between people-space-technology. The adoption of an analytical prism close to and within the social practices in public space can even be an important contribution to answer the many questions that arise in the context of living, thinking and acting in the city. This is because, this approach of 'close and inside' can also be associated to the idea of proximity and assiduity with the study contexts; it makes possible to detect sociocultural regularities and patterns, but it also contributes to overcoming the most visible expressions of the contemporary city, in large part associated with its multiple fragmentations and transformations. Such a perspective collaborates to recognize space as a significant reference for people and on that basis enables us to capture the social daily rhythms of space use and appropriation, and explore how these dynamics define behavioural scenarios, routes, points and landscapes. This, in other words, resonates the importance of micro-geographies (Barker and Shoggen 1973; Wicker 1979; Rapoport 1980; Low 2000a, b). We believe that the role of micro-geographies and the local context are both within the leading factors for urban development on different scales, including national, international or EU level, such as the differences of existing local institutions' policy frameworks could cause differences in the development of urban policy processes (Dukes 2010) and the difference in knowledge on public needs and preferences at the local level, which could lead to difficulties in applicability of modern technologies in public open space, relating to the needs of the public (Radovanova and Radovanov 2017). There is, therefore, a role that ethnography can play in capturing practices, always in transformation, but which define a variety of socio-spatial contexts. This is through the close approach to socio-spatial phenomena, which helps us to capture subtleties and distinctions (Geertz 1973). But, on the other hand, from a set of ethnographic experiences, the analysis should be complemented by a more distanced approach. This contributes to giving meaning to what is not yet part of integrated body of knowledge (cf. Lévi-Strauus, in Magnani 2009: 153).

\section{Cyberpark: What Can Be Analysed from an Ethnographic Perspective?}

An ethnographic approach to the people-space-technology triptych presupposes attention to the three poles, privileging the study of the relationship between them. Rather than considering social actors, spatial contexts and ICTs as separate elements, disseminated and subjugated to a macro and homogeneous urban order, it is essential to explore the dynamics through which the three constituent elements of the triptych are related, generating specific meanings, places and landscapes. 
We are interested in going further from previous analyses in the field which, having an interest in answering certain objectives, do not correspond to what is primarily intended by an ethnographic approach. In the close and inside ethnographical approach, the objective is to provide a framework that contributes to awareness and guide scholars in capturing and understanding the less well-known aspects, though often seemingly obvious, singularities, patterns, etc., of the people-space-technology relationship. From a multidimensional point of view, the ethnography will be of interest to reconcile different levels of interpretation and scales of analysis: from the daily experiences of people in living spaces to those of a socio-historical nature; from social practices, discourses and representations, to aspects of the production and circulation of images and imagery; from planning ideologies to intervention techniques and urban design. In the idealized ethnography design, we suggest here (see Fig. 1) that the relations someone might intend to study, pivot around five linked points or questions of interest: Who are the users? How do they use the space? When do they use the space? Where, i.e. which places do they use? and What do users do (and what artefacts they use) in the spaces?

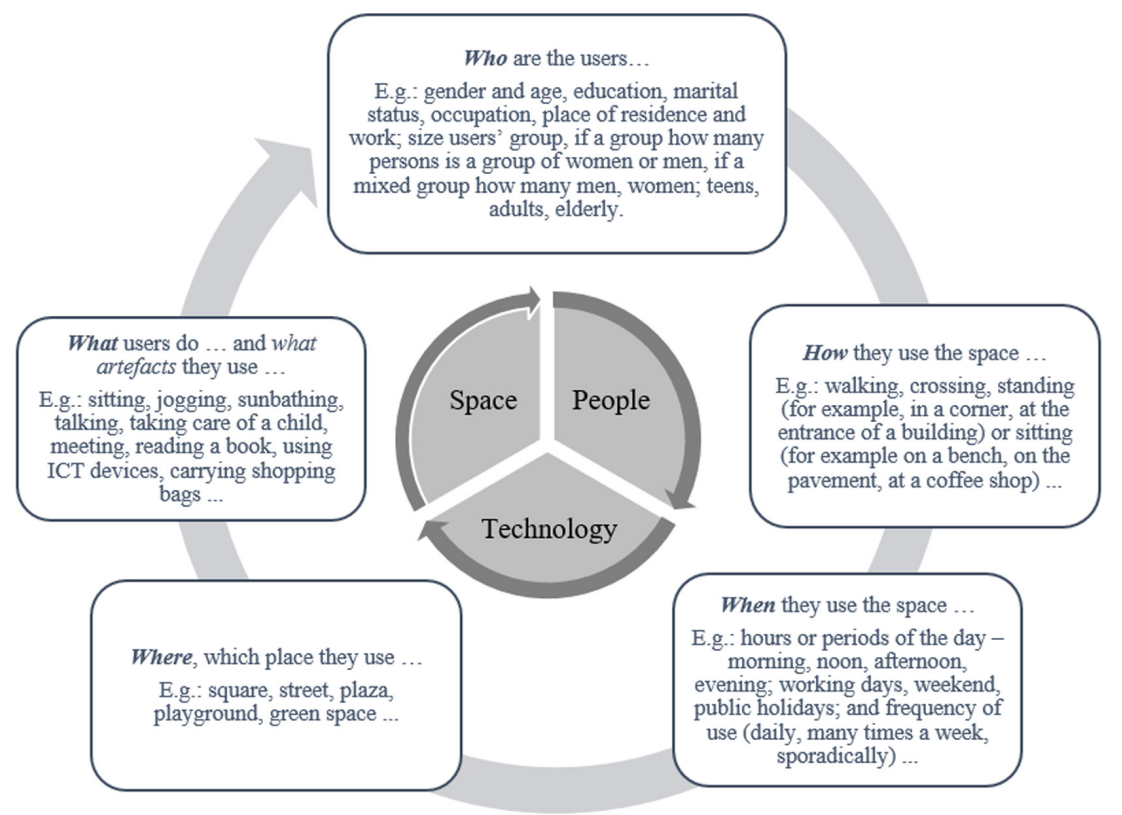

Fig. 1. CyberParks ethnographic approach: capturing the relationship between people-spacetechnology. Source: adapted from Menezes and Smaniotto Costa (2017)

Through ethnographic methods, the aim is to capture the relations between everyday micro-geographies in the use and appropriation of space, the use (or not) of technological artefacts and objects, the practices and socio-spatial representations, and the established relations between spaces, behaviours and environments in the 
constitution of urban places and landscapes (Menezes and Smaniotto Costa 2017) ${ }^{3}$. These relations are still central to understanding the meaning of lived spaces and the constituted spatialities considered important for urban design and planning as envisaged by the CyberParks project.

Having this general framework in mind, Fig. 2 presents the main dimensions research should address providing also the specific content (or objectives) of each dimension. In particular, we argue that the triptych people-space-technology can be ethnologically analysed along three dimensions, all of which keep the human element to the forefront while exploring the nexus of the other two (i.e. POS and ICT). The first dimension concerns the use and appropriation of POS by people.

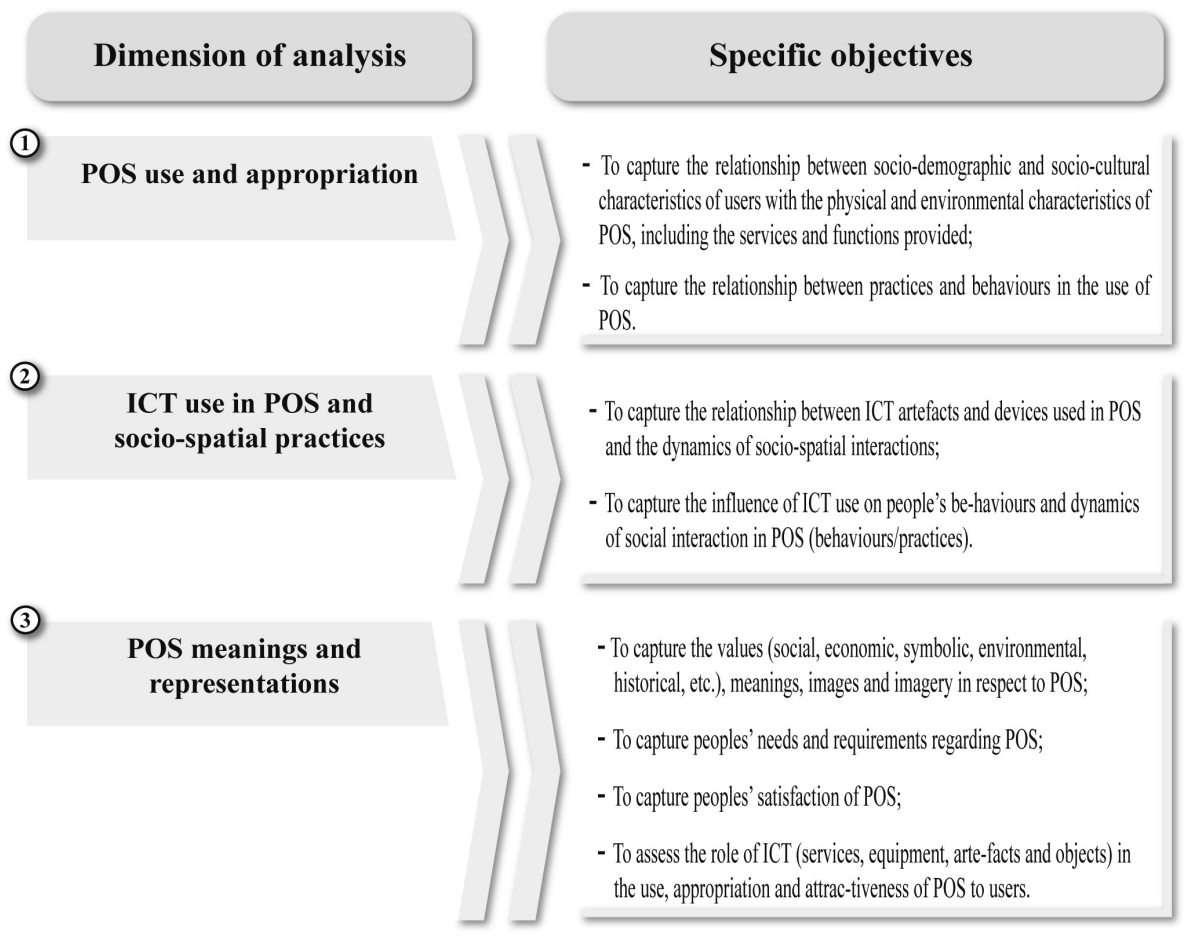

Fig. 2. Dimensions that capture the intersections between POS and ICT. (Objective: to identify the role of people-space-technology aspects of interactions).

Figure 2 focuses on the relationships between the socio-demographic characteristics of the users, on the one hand, and the physical, spatial, environmental and functional characteristics of the POS on the other, and it aims to capture the richness of deployed

${ }^{3}$ Of course, the realization of an ethnography is not alien to the more specific subjects and interests of the researchers, nor to their theoretical and conceptual options, which should be presented and discussed, which then defines the techniques and instruments to be used (and that can be many, for example, observation, interviews, questionnaires, photographs, behavioural maps, etc.). 
behaviours and practices in the use and appropriation of POS. The second dimension brings into play the ICT element, placing emphasis on the way it affects space and social practices. In particular, it focuses on the relationships between ICT usage (of devices, artefacts, etc.) in POS and the dynamics of their interaction, aiming to capture the effect ICTs have on people's behaviour and practises in POS. The last dimension concentrates on meanings and representations of these relations. It aims to capture the richness of values, images and meanings (social, economic, symbolic, environmental, etc.) people attach to POS vis-à-vis their needs and levels of their satisfaction, and to evaluate the contribution of ICT (services, equipment, artefacts, etc.) in the fulfilment of those needs and the enhancement of POS attractiveness to the public.

Having identified the main dimensions of inquiry, the next step is to specify the variables of analysis and the kind of information that needs to be sought. Figures 3, 4 and 5 present these for the first, second and third dimensions respectively. It should be noted that our intention here is not to provide a full check-list of the relevant variables and information required, but rather to eclectically outline indicative aspects in order to convey our rationale and to clarify our perspective.

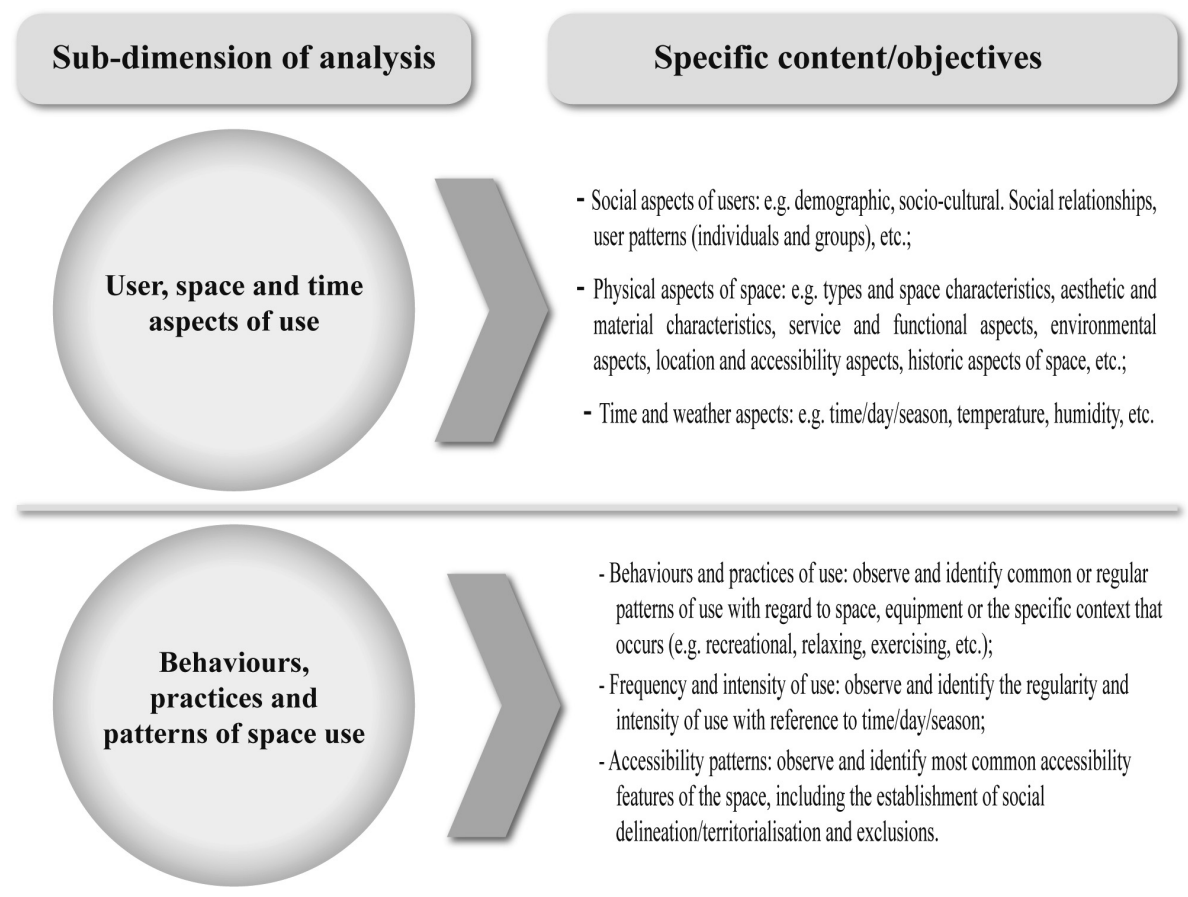

Fig. 3. Aspects that capture POS social use and appropriation (the first dimension).

The framework provided in this chapter has to be seen as holistic view towards public open space development within the scope of "triptych" approach for better understanding people-space-technology relationships. With regard to the first 
dimension of our approach, we envisage two sets of variables that need to be considered (see Fig. 3). The first concentrates on the social aspects of the users and the physical and temporal elements of their POS use. Information that needs to be collected concerns: the socio-demographic characteristics of the users (age, gender, education level, etc.), which provide the profile of the users, socio-cultural attributes (e.g. lifestyle) to allow for a description of the way users use the POS; social relationships (e.g. level of socialisation), which delineate the relationships between users in the POS; and use patterns (e.g. aspects of exclusion or heterogeneity); which outline the ways space is used and appropriated. In regard to the physical aspects of space, one needs to examine the location and accessibility characteristics of the POS, the composition of space (e.g. shadowed-sunny places), its service and functional characteristics (e.g. equipment, facilities and services available) and its environmental elements (vegetation cover, flora and fauna, etc.). The temporal element, finally, concerns the time of the study (hour, day and season) and the weather characteristics associated with it (temperature, humidity, etc.). The second set of variables we suggest concentrates on the practices and behavioural patterns of space usage. These concern the ways that space is used by the visitors (walking, strolling, sitting, exercising, etc.), the pattern of their accessibility, the frequency, regularity and intensity of the use, and the degree of territorialisation that may emerge (e.g. patterns of social or digital exclusion).

\section{Sub-dimension of analysis}

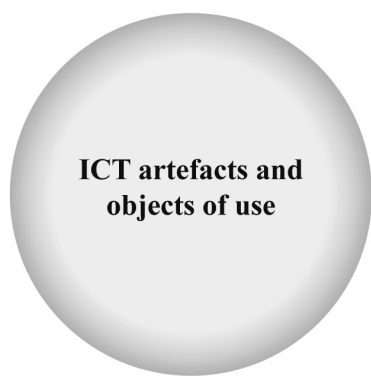

\section{Specific content/objectives}

\footnotetext{
- ICT artefacts and objects used in POS (e.g. computer, smart phone, tablet, etc.);

- Services, equipment and other technological devices provided to support users of ICT (e.g. wi-fi, battery charging stations, etc.);

- Purpose of artefact and object use: observe and identify the reasons for using (or not using) artefacts and objects (e.g. for recreation, communications, social networking, work, shopping, etc.);

- Practices and behaviour patterns of ICT use: observe and identify common or regular social performers and the part of space these occur;

- Frequency and time/duration of ICT use: observe and identify the regularity and intensity of ICT use with reference to duration of use.
}

Fig. 4. Aspects that capture POS-ICT use and socio-spatial practices (the second dimension).

Moving to the second dimension of our framework, we place emphasis on ICT artefacts and objects of use (see Fig. 4). Here researchers need to record the types of devices and applications visitors used (smart phone, laptop, etc.), the artefacts, equipment and facilities the space provides to users (e.g. Wi-Fi access, furniture to electrically charge devices), the purpose of device and artefact use (e.g. for recreation, communication, work), the patterns of such use, and finally, the frequency and timing of the ICT use. 


\section{Sub-dimension of analysis}

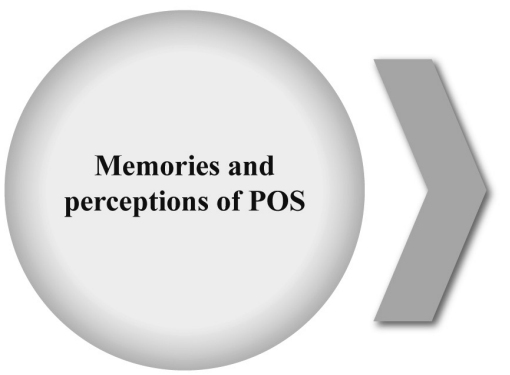

\section{Specific content/objectives}

- Social memories and recordance about space: identify and describe how people interpret their relationship with the POS throughout their lives, and the perceptions influence the use of $\mathrm{POS}$.

- Images and imaginaries in respect to POS: to capture and describe the socio-spatial images conveyed in POS (by media, society, planners, politicians, etc.)

- Perceptions of security and safety.

- Perceptions of quality, e.g. environmental hygiene, noise, air pollution, etc.

- Attractiveness of POS: capture and identify the most and less attractive elements in POS according to social practices, users, physical and environmental aspects, ICT services, etc.

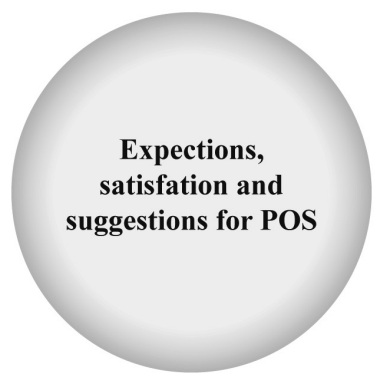

\footnotetext{
- User satisfaction with reference to environmental, physical, social, service and functional aspects (including ICT services), accessibility, security, safety, etc.

- User needs and requeriments: identify of requirements and preferences with reference to social, space, ICT and others important aspects to users.

- Ideas for increase space transformation/improvement: identify suggestions and new ideas to improve the physical, environmental, functional, etc. aspects of space.

- Ideas for ICT transformation/improvement of space: identify suggestions and new ideas to improve the space with technological tools and services; to improve participation and social inclusion in POS.
}

Fig. 5. Aspects that capture the POS social meaning and representations (the third dimension).

In the third dimension of our approach we perceive two key sets of variables that need to be examined (see Fig. 5). The first refers to the memories and perceptions of space, and the second to users' needs and levels of their satisfaction. With regard to the former, information that needs to be collected concerns: the social memories and meanings space connotes (i.e. how people perceive space and their social relationships in space); the images and imaginaries space conveys to different types of users and actors involved (e.g. planners, politicians, etc.); perceptions of security, safety and quality of space (noise, pollution, etc.); and, elements of space attractiveness to users (physical, environmental, digital, social, etc.). Turning to the second set of variables in this dimension, aspects that need to be considered include the level of users' satisfaction from all elements of space (physical, environmental, social, digital, functional, security, etc.), as well as their needs and their requirements. Last, but not least, users could and should provide the researcher with their ideas and suggestions of how space can be improved (in all of its aspects: physical, environmental, functional, etc.) and 
particularly with reference to its digital dimension. This concerns the provision of technological tools, artefacts, facilities and services that would improve POS quality and enhance social inclusion and participation.

\section{Discussion and Conclusions}

Given that a cyber reality has become a tangible dimension of the social world, it makes it more complex to conceptualise, capture and analyse socio-spatial phenomena, namely the social uses and needs of urban public space. This, on the one hand, implies that we need more agile and interdisciplinary approaches to research in this field. On the other hand, the broad dimension, continuous and accelerated growth of the cybernetic phenomenon, in parallel with urban transformation processes, require the production of more focused knowledge (micro-scale) with attention to specific situations and to people. The ethnographic perspective, although not the only available to such kind of inquiry, helps, to a great extent, to analyse and understand all such aspects. This stems from its proper emphasis on the spatio-temporal character of the social phenomena, enabling to formulate and further develop more comprehensive theories. We also strongly argue for the need to continually place people at the centre of our analyses, to best understand the people-space-technology triad. Thus, we advocate for a people-centred approach to research that seeks to understand the ways people use POS, ICTs and engage in a range of social behaviours within such spaces. As such, we feel that the framework presented in this Chapter makes a novel contribution to knowledge in this field and enhances our ability to achieve various goals in our explorations of contemporary understandings of the interactions between people, space and technology. One further aspect of our thinking that remains crucial to any research project, is a recognition that investments in, and developments of, CyberParks, are context specific and that CyberParks occur differently in different geographic locations, and will serve different purposes, as well as have different forms and functions within these locations. The inherent versatility of different locations calls for more call detailed and comprehensive studies to be carried out, as well as comparative research.

In this sense, the ethnographic framework we attempted to assemble in this article can be used for both the development of scientific knowledge on contemporary urban phenomena and for the provision of advice in urban design and planning. Certainly, such a framework cannot incorporate (and definitely we did not intend to do so) all aspects that can (or should) be studied in the context of the relationship between people, space and technology. That would be extremely difficult (if even possible) given the variety of disciplines, theories and perspectives involved, let alone that such an endeavour would be contrary to the spirit of the ethnographic approach itself. Yet, we attempted to pinpoint and incorporate elements we regarded as essential and pertinent to the aspects that emerged during the CyberParks project. What we wish to see is how this framework approach will continue to illustrate the value of cyberparks to different stakeholders, partners and politicians to ensure that it continues to form part of the discussion of planning delivery mechanisms and lead to wider establishment of a modern type of public open space, along with the more traditional forms of landscape investments. 


\section{References}

Barker, R., Shoggen, P.: Qualities of Community Life. Jossey - Bass Publishers, London (1973) Castells, M.: The Internet Galaxy: Reflections on the Internet, Business and Society. Oxford University Press, New York (2001)

Dukes, T.H.: The role of local context: explaining the policy process of the URBAN programmes in Amsterdam and the Hague. Urban Res. Pract. 3(3), 159-176 (2010)

Geertz, C.: The Interpretation of Cultures. Basic Books, New York (1973)

Low, S.M.: On the Plaza - The Politics of Public Space and Culture. University of Texas Press, Austin (2000a)

Low, S.M.: Culture in the modern city: the microgeographies of gender, class, and generation in the Costa Rican plaza. Horiz. Antropol. 6(13), 31-64 (2000b). https://doi.org/10.1590/s010471832000000100003

Low, S.M.: Spatializing Culture: The Ethnography of Space and Place. Routledge, New York (2016)

Magnani, J.G.C.: Etnografia como prática e experiência. Horiz. Antropol. 15(32), 129-156 (2009). https://doi.org/10.1590/S0104-71832009000200006

Menezes, M., Smaniotto Costa, C.: People, public space, digital technology and social practice: an ethnographic approach. In: Zammit, A., Kenna, T. (eds.) Enhancing Places Through Technology, pp. 167-180. Edições Universitárias Lusófonas, Lisbon (2017). ISBN 978-989757-055-1. http://cyberparks-project.eu/sites/default/files/publications/cyberparks_enhancing placestechnology.pdf

Radovanova, P., Radovanov, A.: Urban development in respect to social media - the applicability of the Amsterdam city in other European cities. In: Zammit, A., Kenna, T. (eds.) ICiTy: Enhancing Spaces Through Technology, pp. 129-138. Edições Lusófonas, Lisbon (2017)

Rapoport, A.: Cross-cultural aspects of environmental design. In: Altman, I., Rapoport, A., Wolhwill, J.F. (eds.) Environment and Culture, pp. 7-46. Plenum, New York (1980)

Wicker, A.: An Introduction to Ecological Psychology. Brooks/Cole Publication, San Francisco (1979)

Open Access This chapter is licensed under the terms of the Creative Commons Attribution 4.0 International License (http://creativecommons.org/licenses/by/4.0/), which permits use, sharing, adaptation, distribution and reproduction in any medium or format, as long as you give appropriate credit to the original author(s) and the source, provide a link to the Creative Commons license and indicate if changes were made.

The images or other third party material in this chapter are included in the chapter's Creative Commons license, unless indicated otherwise in a credit line to the material. If material is not included in the chapter's Creative Commons license and your intended use is not permitted by statutory regulation or exceeds the permitted use, you will need to obtain permission directly from the copyright holder.

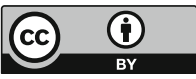

